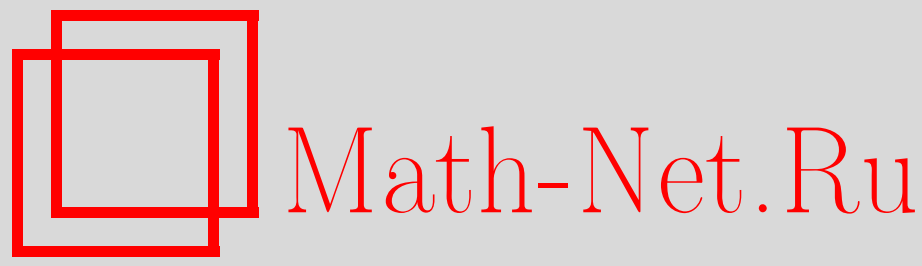

А. А. Славнов, Исследование неоднозначности в неабелевых калибровочных теориях, ТМФ, 2012, том 170, номер 2, 242-247

DOI: https://doi.org/10.4213/tmf6762

Использование Общероссийского математического портала Math-Net.Ru подразумевает, что вы прочитали и согласны с пользовательским соглашением http: //www . mathnet.ru/rus/agreement

Параметры загрузки:

IP: 54.80 .73 .141

26 апреля 2023 г., 10:04:09

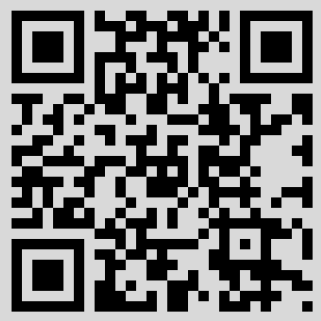




\section{ИССЛЕДОВАНИЕ НЕОДНОЗНАЧНОСТИ В НЕАБЕЛЕВЫХ КАЛИБРОВОЧНЫХ ТЕОРИЯХ}

\footnotetext{
Дан обзор проблемы неоднозначности, возникающей при квантовании неабелевых калибровочных теорий. Предложен метод квантования калибровочных теорий, свободный от неоднозначностей.
}

Ключевые слова: квантование неабелевых теорий, неоднозначность Грибова.

\section{1. ВВЕДЕНИЕ}

В настоящей работе обсуждается проблема неоднозначности, возникающей при квантовании калибровочных теорий. Стандартная формулировка теории ЯнгаМиллса не задает единственной процедуры фиксации калибровки. В работе [1] Грибов показал, что кулоновское калибровочное условие $\partial_{i} A_{i}=0$ не приводит к выбору единственного представителя в классе калибровочно-эквивалентных конфигураций, поскольку условие

$$
\partial_{i} A_{i}^{\Omega}=0
$$

рассматриваемое как уравнение относительно элементов калибровочной группы $\Omega$ на поверхности $\partial_{i} A^{i}=0$, при достаточно больших $A$ имеет нетривиальные решения, быстро спадающие на пространственной бесконечности. Этот результат был обобщен в работе [2] на произвольные ковариантные калибровочные условия.

В рамках теории возмущений, т. е. при достаточно малых $A$, уравнение (1) допускает только тривиальные решения, поэтому неоднозначность Грибова в этом случае отсутствует. Тем не менее вне рамок теории возмущений неоднозначность Грибова имеет место, что делает проблематичным стандартный подход канонического квантования неабелевых калибровочных теорий.

Можно попытаться избежать этой неоднозначности, если применить так называемые алгебраические калибровочные условия, например выбрать гамильтонову калибровку $A_{0}=0$. Однако такое калибровочное условие явно нарушает лоренцеву инвариантность, что катастрофически усложняет анализ. Оно также приводит к другим проблемам, которые здесь не рассматриваются.

*Математический институт им. В. А. Стеклова РАН, Москва, Россия. E-mail: slavnov@mi.ras.ru 
В соответствии с общепринятыми представлениями относительно квантования неабелевых калибровочных теорий имеет место дилемма: дифференциальные калибровочные условия типа лоренцевой калибровки $\partial_{\mu} A_{\mu}=0$ испорчены неоднозначностью Грибова, а алгебраические калибровочные условия приводят к потере явной лоренцевой инвариантности, а потому к серьезным проблемам при перенормировке теории. Возможный выход из создавшейся ситуации связан с новой формулировкой неабелевых калибровочных теорий, в которой вводится большее число вспомогательных полей. Пример такой теории дается моделью Хиггса, описываемой лагранжианом

$$
L=L_{\mathrm{YM}}+\left(D_{\mu} \varphi\right)^{*}\left(D_{\mu} \varphi\right)-\lambda^{2}\left(\varphi^{*} \varphi-\mu^{2}\right)^{2},
$$

где $L_{\mathrm{YM}}$ - обычный лагранжиан Янга-Миллса. После сдвига поле $\varphi=\varphi^{\prime}+\hat{\mu}$, где $\hat{\mu}=\{0, \mu\}$, превращается в калибровочное поле $\varphi_{a}^{\prime} \rightarrow \varphi_{a}^{\prime}+\mu \eta^{a}(x)+\cdots, a=1,2,3$. Унитарная калибровка $\varphi_{a}^{\prime}=0$ при этом оказывается алгебраической и одновременно лоренц-инвариантной. При этом скаляр Хиггса задается не одним полем, а четырехкомпонентным $S U(2)$-спинором, три нефизических компоненты которого должны быть убраны с помощью калибровки. Унитарная калибровка тем не менее оказывается неперенормируемой.

Можно применить похожую идею для формулировки свободной от неоднозначностей перенормируемой теории Янга-Миллса. Автором недавно была предложена явно лоренц-инвариантная формулировка квантовой теории Янга-Миллса, в которой эффективный лагранжиан полей ду́хов оказывается калибровочно-инвариантным [3]. В настоящей работе показано, что для теории Янга-Миллса при данном подходе возможна процедура квантования, свободная от неоднозначностей Грибова, которая тем самым может служить отправной точкой при построении непертурбативных конструкций [4]. В рамках теории возмущений модель оказывается перенормируемой, хотя процедура перенормировки включает в себя немультипликативное переопределение полей [5].

\section{2. КВАНТОВАНИЕ ПОЛЯ ЯНГА-МИЛЛСА, СВОБОДНОЕ ОТ НЕОДНОЗНАЧНОСТЕЙ}

Рассмотрим модель, описываемую классическим лагранжианом

$$
L=-\frac{1}{4} F_{\mu \nu}^{a} F_{\mu \nu}^{a}+\left(D_{\mu} \varphi\right)^{*}\left(D_{\mu} \varphi\right)-\left(D_{\mu} \chi\right)^{*}\left(D_{\mu} \chi\right)+i\left[\left(D_{\mu} b\right)^{*}\left(D_{\mu} e\right)-\left(D_{\mu} e\right)^{*}\left(D_{\mu} b\right)\right] .
$$

Чтобы не слишком усложнять изложение, рассмотрим модель с калибровочной группой $S U(2)$. Эту модель нетрудно обобщить на другие калибровочные группы. В соотношении (2) $F_{\mu \nu}^{a}$ представляет собой стандартный тензор напряжений поля Янга-Миллса. Скалярные поля $\varphi, \chi, b, e$ образуют комплекснозначные $S U(2)$-дублеты, параметризованные эрмитовыми компонентами следующим образом:

$$
c=\left(\frac{i c_{1}+c_{2}}{\sqrt{2}}, \frac{c_{0}-i c_{3}}{\sqrt{2}}\right),
$$

где $c$ означает любой из дублетов. Поля $\varphi$ и $\chi$ коммутируют, а поля $e$ и $b$ антикоммутируют между собой. Символ $D_{\mu}$ обозначает обычную ковариантную производную, благодаря чему лагранжиан $L$ оказывается калибровочно-инвариантным. Заметим, 
что, поскольку лагранжиан поля $\chi$ входит с отрицательным знаком, энергия этого поля отрицательна.

Произведем следующий сдвиг в лагранжиане (2):

$$
\varphi \rightarrow \varphi+g^{-1} \widehat{m}, \quad \chi \rightarrow \chi-g^{-1} \widehat{m},
$$

где $\widehat{m}=(0, m)$ и $m$ - постоянный параметр. Благодаря отрицательному знаку лагранжиана поля $\chi$ члены, квадратичные по $m$, которые возникают при сдвигах полей $\varphi$ и $\chi$, взаимно сокращаются, и лагранжиан принимает вид

$$
\begin{aligned}
L=\frac{1}{4} & F_{\mu \nu}^{a} F_{\mu \nu}^{a}+\left(D_{\mu} \varphi\right)^{*}\left(D_{\mu} \varphi\right)-\left(D_{\mu} \chi\right)^{*}\left(D_{\mu} \chi\right)+ \\
& +g^{-1}\left[\left(D_{\mu} \varphi\right)^{*}+\left(D_{\mu} \chi\right)^{*}\right]\left(D_{\mu} \widehat{m}\right)+g^{1}\left(D_{\mu} \widehat{m}\right)^{*}\left[D_{\mu} \varphi+D_{\mu} \chi\right]+ \\
& +i\left[\left(D_{\mu} b\right)^{*}\left(D_{\mu} e\right)-\left(D_{\mu} e\right)^{*}\left(D_{\mu} b\right)\right] .
\end{aligned}
$$

Как и прежде, этот лагранжиан описывает безмассовые векторные частицы.

Лагранжиан (3), очевидно, инвариантен относительно “сдвинутых" калибровочных преобразований. В частности, преобразование поля $\varphi_{-}^{a}=(\varphi-\chi) / \sqrt{2}$ имеет вид

$$
\delta \varphi_{-}^{a}=m \eta^{a}+\frac{g}{2} \varepsilon^{a b c} \varphi_{-}^{b} \eta^{c}+\frac{g}{2} \varphi_{-}^{0} \eta^{a} .
$$

Кроме того что лагранжиан (3) калибровочно-инвариантен, он также инвариантен относительно преобразований суперсимметрии

$$
\begin{aligned}
\delta \varphi(x) & =i \epsilon b(x), & \delta \chi(x) & =-i \epsilon b(x), \\
\delta e(x) & =\epsilon[\varphi(x)+\chi(x)], & \delta b(x) & =0,
\end{aligned}
$$

в которых $\epsilon$ - постоянный антикоммутирующий параметр.

Далее показано, что инвариантность относительно преобразований суперсимметрии обеспечивает унитарность теории в пространстве, которое состоит только из физических возмущений полей. Явный вид членов взаимодействия при этом не играет роли, важными оказываются только свойства симметрии. В принципе разрешены произвольные контрчлены, которые сохраняют одновременно калибровочную инвариантность и суперсимметрию.

При калибровочном преобразовании поле $\varphi_{-}^{a}$ сдвигается на произвольную функцию $m \eta^{a}$. Это позволяет наложить лоренц-инвариантное алгебраическое калибровочное условие $\varphi_{-}^{a}=0$. Тем не менее само по себе наложение такого условия не полностью решает проблему неоднозначности. Поле $\varphi_{-}^{a}$, удовлетворяющее условию $\varphi_{-}^{a}=0$, переходит при калибровочном преобразовании в поле $\varphi_{-}^{\prime a}=\left(m+g \varphi_{-}^{0} / 2\right) \eta^{a}$. При некоторых $x$ множитель $m+g \varphi_{-}^{0}(x) / 2$ может обращаться в нуль, что может привести к неоднозначности при фиксации калибровки. Чтобы полностью устранить проблему неоднозначности, переопределим поля следующим образом:

$$
\begin{aligned}
\varphi_{-}^{0} & =\frac{2 m}{g}\left(e^{g h / 2 m}-1\right), & \varphi_{-}^{a} & =\widetilde{M} \tilde{\varphi}_{-}^{a}, \\
\varphi_{+}^{0} & =\widetilde{M}^{-1} \tilde{\varphi}_{+}^{0}, & \varphi_{+}^{a} & =\widetilde{M} \\
e & =\widetilde{M}^{-1} \tilde{e} \tilde{\varphi}_{+}^{a}, & b & =\widetilde{M} \tilde{b},
\end{aligned}
$$


где

$$
\widetilde{M}=1+\frac{g}{2 m} \varphi_{-}^{0}=e^{g h / 2 m} .
$$

Новый лагранжиан имеет при этом вид

$$
\begin{aligned}
\tilde{L}= & -\frac{1}{4} F_{\mu \nu}^{a} F_{\mu \nu}^{a}+\partial_{\mu} h \partial_{\mu} \tilde{\varphi}_{+}^{0}-\frac{g}{2 m} \partial_{\mu} h \partial_{\mu} h \tilde{\varphi}_{+}^{0}+m \tilde{\varphi}_{+}^{a} \partial_{\mu} A_{\mu}^{a}- \\
& -\left[\left(\left(D_{\mu} \tilde{b}\right)^{*}+\frac{g}{2 m} \tilde{b}^{*} \partial_{\mu} h\right)\left(D_{\mu} \tilde{e}-\frac{g}{2 m} \tilde{e} \partial_{\mu} h\right)+\text { э.c. }\right]+ \\
& +\frac{m g}{2} A_{\mu}^{2} \tilde{\varphi}_{+}^{0}+g \partial_{\mu} h A_{\mu}^{a} \tilde{\varphi}_{+}^{a}+\cdots,
\end{aligned}
$$

где многоточие стоит вместо членов, пропорциональных $\tilde{\varphi}_{-}^{a}$. По построению этот лагранжиан инвариантен относительно калибровочных преобразований, записанных в терминах новых переменных. В частности, имеем $\delta \tilde{\varphi}_{-}^{a}=\eta^{a}$, и неоднозначность не имеет места.

Построенный лагранжиан, очевидно, инвариантен относительно преобразований суперсимметрии, записанных в терминах сдвинутых переменных. Но наложение калибровочного условия $\tilde{\varphi}_{-}^{a}=0$ нарушает инвариантность эффективного действия относительно преобразования суперсимметрии (4). Переход от одной калибровки к другой можно осуществить с помощью калибровочного преобразования, и в калибровке $\partial_{i} A_{i}=0$ эффективное действие оказывается инвариантным относительно преобразования суперсимметрии (4), поэтому действие в калибровке $\tilde{\varphi}_{-}^{a}=0$ также должно быть инвариантным относительно некоторого преобразования суперсимметрии. Соответствующая калибровочная функция оказывается решением уравнения

$$
\int \lambda^{a}(x) \partial_{i}\left(A^{\Omega}\right)_{i}^{a}(x) d^{4} x=\int \lambda^{a}(x) \tilde{\varphi}_{-}^{a}(x) d^{4} x .
$$

Решение этого уравнения можно найти в явном виде.

Эффективный лагранжиан (5) инвариантен относительно вышеприведенных преобразований суперсимметрии, равно как и относительно преобразований глобальной $S U(2)$-симметрии, которые не затрагивают поля $\tilde{\varphi}_{ \pm}^{0}$ и $\tilde{\varphi}_{-}^{a}$.

Спектр теории состоит из следующих полей: возбуждения, описываемые полями ду́хов $\varphi_{ \pm}, b, e$, продольные и временны́е компоненты полей $A_{\mu}^{a}$. Физические возбуждения суть три трехмерно поперечные компоненты поля Янга-Миллса. Преобразование суперсимметрии эффективного действия задает сохраняющийся нильпотентный заряд $Q$. Физические состояния выделяются условием $Q|\psi\rangle_{\mathrm{ph}}=0$. Состояния, выделенные этим условием, включают в себя только три трехмерно поперечных компоненты поля Янга-Миллса. Возбуждения, описываемые полями ду́хов, отщепляются.

\section{3. ПЕРЕНОРМИРОВКА}

Поле $h$ входит в члены взаимодействия только в виде производной $\partial_{\mu} h$, поэтому индекс расходимости диаграммы с $n$ внешними линиями поля $h\left(\varphi_{-}^{0}\right)$ уменьшается на $n$. Индекс расходимости произвольной диаграммы равен

$$
n=4-2 L_{\varphi_{+}^{0}}-2 L_{\varphi_{+}^{0}}-L_{A}-L_{e}-L_{b}-L_{h} .
$$


Теория тем самым оказывается явно перенормируемой. В терминах старых (непреобразованных) переменных теория не обладает свойством явной перенормируемости. Переход к новым переменным одновременно устраняет остаточную неоднозначность и делает теорию явно перенормируемой. Перенормировка сохраняет все симметрии теории. Возможные контрчлены можно классифицировать на основе тождеств Славнова-Тейлора, которые связаны с симметрией, объединяющей калибровочную инвариантность эффективной теории и суперсимметрию:

$$
S(\Gamma)=\int \sum_{\Phi}\left\{\frac{\delta \Gamma}{\delta \Phi^{*}(x)} \frac{\delta \Gamma}{\delta \Phi(x)}\right\} d x^{4}=0 .
$$

Поля $\Phi$ в этом выражении суть $A_{\mu}, \varphi_{+}^{\alpha}, e^{\alpha}, b^{\alpha}$. Поля $\Phi^{*}$ представляют собой антиполя, соответствующие вариациям полей $\Phi$, например

$$
\int\left\{-\frac{2 i}{m} A_{\mu}^{* a}\left(D_{\mu} b\right)^{a}\right\} d x .
$$

Наиболее общее решение тождеств Славнова-Тейлора, совместное с анализом степеней расходимости и с остаточной $S U(2)$-симметрией, имеет следующую структуру. В классическом действии перенормировка затрагивает параметры $g^{\prime}=Z_{g} g$ и $m^{\prime}=Z_{m} m$, при этом производится переопределение полей

$$
\begin{gathered}
\tilde{e}^{\prime}=Z_{1} \tilde{e}, \quad \tilde{b}^{\prime}=Z_{m} \tilde{b}, \quad A_{\mu}^{a^{\prime}}=Z_{2} A_{\mu}^{a}, \quad h^{\prime}=Z_{m} Z_{3} h, \\
\tilde{\varphi}_{+}^{a^{\prime}}=Z_{4} \tilde{\varphi}_{+}^{a}+Z_{5} \partial_{\mu} A_{\mu}^{a}+\frac{Z_{6}}{m} \partial_{\mu} h A_{\mu}^{a}+Z_{7}\left(\tilde{e}^{0} \tilde{b}^{a}-\tilde{e}^{a} \tilde{b}^{0}-\varepsilon^{a b c} \tilde{e}^{b} \tilde{b}^{c}\right), \\
\tilde{\varphi}_{+}^{0^{\prime}}=Z_{8} \tilde{\varphi}_{+}^{0}+Z_{9} \frac{\partial^{2}}{m} h+Z_{10} \frac{1}{m^{2}} \partial_{\mu} h \partial_{\mu} h+Z_{11} A^{2}+Z_{12}\left(\tilde{e}^{0} \tilde{b}^{0}+\tilde{e}^{a} \tilde{b}^{a}\right) .
\end{gathered}
$$

Чтобы удовлетворить тождествам Славнова-Тейлора (6), также необходимо переопределить антиполя. Соответствующие уравнения допускают явное решение.

Перенормированное действие отличается от неперенормированного только перенормировкой параметров, которые входят в неперенормированное эффективное действие, и переопределением полей (7):

$$
\Gamma_{\mathrm{R}}\left(g^{\prime}, m^{\prime}, \Phi^{\prime}\right)=\Gamma_{\mathrm{cl}}\left(Z_{g} g, Z_{m} m, \Phi\left(\Phi^{\prime}\right)\right)
$$

Все ультрафиолетовые расходимости могут быть последовательно устранены путем подходящего выбора параметров $Z_{g}, Z_{m}, Z_{j}$.

\section{4. КАЛИБРОВОЧНАЯ ИНВАРИАНТНОСТЬ НАБЛЮДАЕМЫХ ВЕЛИЧИН}

Все переопределения полей локальны и не затрагивают поле $\tilde{\varphi}_{-}^{a}$, поэтому в предположении существования инвариантной регуляризации типа размерной, производя обратное переопределение, получим для матрицы рассеяния представление в виде интеграла по путям:

$$
S=\int \exp \left\{i \int\left[L_{\mathrm{gi}}+\lambda^{a} \varphi_{-}^{a}\right] d x\right\} \operatorname{det}\left(M_{a b}\right) d \mu,
$$


где $L_{\mathrm{gi}}$ представляет собой инвариантный классический лагранжиан с перенормированными параметрами и

$$
\left.\left(\operatorname{det}\left(M_{a b}\right)\right)^{-1}\right|_{\varphi_{-}^{a}=0}=\int \delta\left(\left(\varphi^{\Omega}\right)_{-}^{a}\right) d \Omega
$$

Умножим интеграл (8) на "единицу" $1=\Delta_{\mathrm{L}} \int \delta\left(\partial_{\mu} A_{\mu}^{\Omega}\right) d \Omega$ и произведем замену переменных $\Phi^{\Omega}=\Phi^{\prime}$, в результате получим выражение для матрицы рассеяния в лоренцевой калибровке

$$
S=\int \exp \left\{i \int\left[L_{\mathrm{gi}}(x)+\lambda^{a}(x) \partial_{\mu} A_{\mu}(x)+\partial_{\mu} \bar{c}^{a}\left[D_{\mu} c\right]^{a}\right] d x\right\} d \mu,
$$

которое нетрудно преобразовать к стандартному выражению для матрицы рассеяния полей Янга-Миллса.

\section{5. ЗАКЛЮЧЕНИЕ}

Продемонстрирована возможность построения перенормируемой и явно лоренцинвариантной формулировки теории Янга-Миллса, которая допускает каноническое квантование, свободное от неоднозначностей Грибова. В теории возмущений (инфракрасно регуляризованная) матрица рассеяния и калибровочно-инвариантные корреляционные функции полей Янга-Миллса совпадают со стандартными. Аналогичный формализм можно развить и в случае модели Хиггса-Киббла, описывающей массивное поле Янга-Миллса и массивный скаляр Хиггса. В этом случае инфракрасные расходимости отсутствуют, и матрица рассеяния оказывается корректно определенной. Как и в случае безмассовой теории Янга-Миллса, эта модель допускает модифицированную формулировку, в которой можно наложить свободное от неоднозначностей калибровочное условие, сохраняющее явную лоренц-инвариантность и перенормируемость теории. Представляется интересным произвести полуаналитические и численные расчеты в рамках этого формализма, не привлекая теорию возмущений, и сравнить полученные при этом результаты с результатами уже проведенных вычислений.

Благодарности. Работа частично поддержана РФФИ (грант № 09-01-12150).

\section{Список литературы}

[1] V. N. Gribov, Nucl. Phys. B, 139:1-2 (1978), 1-19.

[2] I. M. Singer, Commun. Math. Phys., 60:1 (1978), 7-12.

[3] A. A. Slavnov, JHEP, 08 (2008), 047, 10 pp., arXiv: 0807.1795.

[4] А. А. Славнов, ТМФ, 154:2 (2008), 213-219.

[5] A. Quadri, A. A. Slavnov, JHEP, 07 (2010), 087, 22 pp., arXiv: 1002.2490. 